\title{
Threshold Dynamics of an SEIS Epidemic Model with Nonlinear Incidence Rates
}

\author{
Mouhcine Naim ${ }^{1} \cdot$ Fouad Lahmidi ${ }^{1} \cdot$ Abdelwahed Namir $^{2}$
}

Accepted: 4 September 2021

(c) Foundation for Scientific Research and Technological Innovation 2021

\begin{abstract}
In this paper, we consider an SEIS epidemic model with infectious force in latent and infected period, which incorporates by nonlinear incidence rates. The local stability of the equilibria is discussed. By means of Lyapunov functionals and LaSalle's invariance principle, we proved the global asymptotic stability of the disease-free equilibrium and the endemic equilibrium. An application is given and numerical simulation results based on real data of COVID-19 in Morocco are performed to justify theoretical findings.
\end{abstract}

Keywords SEIS epidemic model · COVID-19 · Latent period · Local stability · Global stability

\section{Introduction}

In our life there are various infectious diseases that we face, such as coronavirus (COVID19) that was first identified in the Chinese city of Wuhan in 2019, and it is a new strain that has not been previously identified in humans. COVID-19 is a fatal illness caused by a severe acute respiratory syndrome that is transmitted from human-to-human [1]. In epidemiology, mathematical models describing the population dynamics of infectious diseases plays an important role in analyzing the spread and controlling these diseases.

Some diseases, such as tuberculosis, measles, AIDS, COVID-19, have a latent or incubation period when a susceptible has become infected but not yet infectious. COVID-19, for example, has about 5-6 days latent period [2] . This latency can be modeled by introducing a new class, called the exposed class, in which the susceptible remains for a given length of time before moving into the infective class. The resulting models are of $S E I$, $S E I S, S E I R, S E I R S$, or SEIRI type (here, $S, E, I$ and $R$ denote the numbers of susceptible, exposed, infectious and recovered individuals, respectively), see, e.g., [3-8].

Mouhcine Naim

naimmouhcine2013@gmail.com

1 Laboratory of Analysis, Modeling and Simulation, Faculty of Sciences Ben M'sik, Hassan II University, P.O. Box 7955, Casablanca, Sidi Othman, Morocco

2 Laboratory of Information Technology and Modeling, Faculty of Sciences Ben M'sik, Hassan II University, P.O. Box 7955, Casablanca, Sidi Othman, Morocco 
In many diseases the infection is not only in the infected period but even in the latent period, such as COVID-19 at present [9]. Many epidemic models with the infectious force in the latent period have been performed. Yuang et al. in [10] considered the local stability of an epidemic model having infectious force in both latent period and infected period. Guihua and Zhen $[11,12]$ studied the global stability of an SEI epidemic model having infectious force in both latent period and infected period. Global stability of an SEIS epidemic model with standard incidence rate having infectious force in both latent period and infected period given by Mukhopadhyay and Bhattacharyya [13]. The method used in [11-13] for proving global asymptotic stability of epidemic equilibrium is based on the geometric approaches developed by Smith [14], Li and Muldowney [15].

The incidence of a disease is the number of new cases per unit time and plays an important role in the modelling of epidemic dynamics. In many epidemic models, the bilinear incidence rate $\beta S I$ ( $\beta$ denote the transmission rate, which measures the infection force of the disease) $[16,17]$ and the standard incidence rate $\frac{\beta S I}{N}$, where $N$ is the total population size [18], are frequently used. However, the bilinear incidence rate may face many problems and challenges when it is used to describe the evolution of infectious diseases among human. Consequently, the bilinear incidence rate should be modified into a nonlinear incidence rate which play a vital role in ensuring that the model can give a reasonable qualitative description of the capture the nonlinear nature of disease propagation law. Initially, Capasso and Serio [19] in the study of the cholera epidemic spread in Bari in 1973, observed that the incidence rate may increase more slowly as $I$ increases rather than linearly increasing, and found the saturated incidence rate $\frac{\beta S I}{1+\alpha I}$ which gives good agreement with observations, where the parameter $\alpha$ measures the inhibitory effect either due to the crowding caused by high numbers of cases or from the behavioral change of susceptible individuals as their number increases. From then on, a number of authors have studied epidemiological models with several nonlinear incidence rates, see, e.g., [20-26].

In this paper we will consider an SEIS epidemic model with general incidence rates that the diseases such as COVID-19 can be infected in the latent period and the infected period. Hence, our study will be carried out on the following epidemic model:

$$
\left\{\begin{array}{l}
\frac{d S(t)}{d t}=\Lambda-\mu S(t)-S(t) f_{1}(I(t))-S(t) f_{2}(E(t))+\gamma I(t) \\
\frac{d E(t)}{d t}=S(t) f_{1}(I(t))+S(t) f_{2}(E(t))-(\mu+\sigma) E(t) \\
\frac{d I(t)}{d t}=\sigma E(t)-(\mu+\delta+\gamma) I(t)
\end{array}\right.
$$

where $\Lambda$ is the recruitment rate of the susceptible, $\mu$ is the natural death rate of the population, $\sigma$ is the transfer rate from the exposed group to the infectious group so that $\frac{1}{\sigma}$ is the mean latent period, $\delta$ is the death rate due to disease, $\gamma$ is the transfer rate from the infectious group to the susceptible group. In this work, we assume that $\delta$ and $\gamma$ are nonnegative constants, and $\Lambda, \mu$, and $\sigma$ are positive constants.

To study the dynamics of the model (1), we suppose that the functions $f_{i}(i=1,2)$ are continuously differentiable on $[0,+\infty)$ satisfying the following two hypotheses.

(H1) $f_{i}(x)$ is strictly monotone increasing on $[0,+\infty)$ with $f_{i}(0)=0$.

(H2) $\frac{f_{i}(x)}{x}$ is monotone decreasing on $(0,+\infty)$. 
The hypothesis $(H 1)$ is a basic requirement for any biologically feasible incidence rate, since it is obvious that the disease cannot spread when the number of exposed and infected individuals is zero. In addition, the incidence $S f_{1}(I)$ (resp., $S f_{2}(E)$ ) becomes faster as the number of the infectious (resp., exposed) individuals increases.

Since the incidence $S f_{1}(I)$ (resp., $\left.S f_{2}(E)\right)$ can be rewritten as $\frac{f_{1}(I)}{I} S I$ (resp., $\frac{f_{2}(E)}{E} S E$ ). Then, function $\frac{f_{1}(I)}{I}$ (resp., $\frac{f_{2}(E)}{E}$ ) refers to effective contact rate between an infective (resp., exposed) and a susceptible individuals. The hypothesis (H2) implies that the effective contact rate between infective (resp., exposed) and susceptible individuals is decreasing with increase of infectives since the individuals tend to reduce the number of contacts among them per unit time when they are under intervention policies. The idea of hypothesis $(H 2)$ has first been explored in [19].

By the hypotheses $(H 1)-(H 2)$, it can be seen that $f_{i}$ satisfying

$$
0<\frac{f_{i}(x)}{x} \leq f_{i}^{\prime}(0) \text { for all } x>0
$$

and consequently, the effective contact rates are bounded.

The functions $f_{i}(i=1,2)$ includes a number of especial incidence rates. For instance:

1. $f_{i}(x)=\frac{\beta_{i} x}{1+\alpha_{i} x^{p_{i}}}, \alpha_{i} \geq 0,0<p_{i} \leq 1[27]$.

2. $f_{i}(x)=\left(\beta_{i}-\frac{\sigma_{i} x}{m_{i}+x}\right) x, \beta_{i}>\sigma_{i}, m_{i}>0[28,29]$.

On the positivity and boundedness of solutions, we have the following result. Since the proof is simple, we here omit it.

Proposition 1 Let $(S(t), E(t), I(t))$ be any solution of system (1) with any nonnegative initial condition, then $S(t) \geq 0, E(t) \geq 0$ and $I(t) \geq 0$ for all $t \geq 0$. In addition, we have $\limsup _{t \rightarrow+\infty}(S(t)+E(t)+I(t)) \leq \frac{\Lambda}{\mu}$.

The rest of this paper is organized as follows. In the next section, we discuss the existence and the local stability of the equilibria. In Section 3, by using Lyapunov functionals and LaSalle's invariance principle, we establish the global asymptotic stability of the equilibria. An application and illustrative numerical simulations have been given in Section 4, and the numerical results of real data of COVID-19 in Morocco is conducted in the same section. Finally, conclusion is given in Section 5.

\section{Equilibria and Their Local Stability}

In this section, we discuss the existence and the local stability of the equilibria of system (1). It is easy to visualize that the system (1) always has a disease-free equilibrium $E_{0}=\left(S_{0}, 0,0\right)$, where $S_{0}=\frac{\Lambda}{\mu}$, that is, there is no infection present in the population and all individuals are susceptible.

By applying the next generation matrix approach provided by van den Driessche and Watmough [30], the basic reproduction number of model (1) is defined as follows

$$
R_{0}=S_{0} \frac{\sigma f_{1}^{\prime}(0)+\omega_{2} f_{2}^{\prime}(0)}{\omega_{1} \omega_{2}},
$$


where $\omega_{1}=\mu+\sigma$ and $\omega_{2}=\mu+\delta+\gamma$. Biologically, $R_{0}$ represents the average number of secondary infectious cases produced by an exposed individual and an infectious individual during their effective infectious period when introduced in a completely susceptible population [31].

Now, we obtain the following lemma which gives the result on the unique existence of the endemic equilibrium $E_{*}$ for $R_{0}>1$.

Lemma 1 System (1) has a unique positive endemic equilibrium $E_{*}=\left(S^{*}, E^{*}, I^{*}\right)$ if and only if $R_{0}>1$.

Proof Assume that $R_{0}>1$. Suppose that $(S, E, I)$ is any positive equilibrium of system (1), then

$$
\left\{\begin{array}{l}
\Lambda-\mu S-S f_{1}(I)-S f_{2}(E)+\gamma I=0 \\
S f_{1}(I)+S f_{2}(E)-\omega_{1} E=0 \\
\sigma E-\omega_{2} I=0 .
\end{array}\right.
$$

From the third equation of system (3), we have

$$
E=\frac{\omega_{2}}{\sigma} I,
$$

and by summing all equations of system (3), we find that

$$
S=\frac{\Lambda-\phi I}{\mu},
$$

where $\phi=\frac{\mu \omega_{2}}{\sigma}+\mu+\delta$. We have $S \geq 0$ implies that $I \in\left(0, \frac{\Lambda}{\phi}\right]$. Hence, no positive equilibrium exists if $I \geq \frac{\Lambda}{\phi}$. On the other hand, by the second equation of (3) and equation (4), it follows that

$$
S=\frac{\omega_{1} \omega_{2}}{\sigma} \frac{1}{\frac{f_{1}(I)}{I}+\frac{f_{2}\left(\frac{\omega_{2}}{\sigma} I\right)}{I}} .
$$

Then, by equations (5) and (6), $I$ is a positive zero of the function $F$, where

$$
F(I)=\Lambda-\phi I-\frac{\mu \omega_{1} \omega_{2}}{\sigma} \frac{1}{\frac{f_{1}(I)}{I}+\frac{f_{2}\left(\frac{\omega_{2}}{\sigma} I\right)}{I}}, I \in\left(0, \frac{\Lambda}{\phi}\right] .
$$

By the hypothesis $(H 2)$, the function $F$ is strictly monotone decreasing on $\left(0, \frac{\Lambda}{\phi}\right]$ satisfying

$$
\lim _{I \rightarrow 0^{+}} F(I)=\Lambda-\frac{\mu \omega_{1} \omega_{2}}{\sigma} \frac{1}{f_{1}^{\prime}(0)+\frac{\omega_{2}}{\sigma} f_{2}^{\prime}(0)}=\Lambda\left(1-\frac{1}{R_{0}}\right)>0,
$$

and $F\left(\frac{\Lambda}{\phi}\right)<0$. Hence, there is a unique $I^{*} \in\left(0, \frac{\Lambda}{\phi}\right)$ such that $F\left(I^{*}\right)=0$ if $R_{0}>1$. This shows that model (1) has a unique positive endemic equilibrium $E_{*}=\left(S^{*}, E^{*}, I^{*}\right)$ when 
$R_{0}>1$, where $E^{*}$ and $S^{*}$ are determined by (4) and (5), respectively. Second we assume $R_{0} \leq 1$. Then it is obvious that system (1) has no endemic equilibria. Hence the proof is complete.

Next, we focus on the local stability of the disease-free equilibrium $E_{0}$. The Jacobian matrix of system (1) at the equilibrium $E_{0}$ is as follows

$$
J_{E_{0}}=\left(\begin{array}{ccc}
-\mu & -S_{0} f_{2}^{\prime}(0) & -S_{0} f_{1}^{\prime}(0)+\gamma \\
0 & S_{0} f_{2}^{\prime}(0)-\omega_{1} & S_{0} f_{1}^{\prime}(0) \\
0 & \sigma & -\omega_{2}
\end{array}\right) .
$$

Clearly, $\lambda_{1}=-\mu<0$ is a eigenvalue of $J_{E_{0}}$. The other two eigenvalues of $J_{E_{0}}$ are determined by the following equation

$$
\lambda^{2}+\left(\omega_{1}+\omega_{2}-S_{0} f_{2}^{\prime}(0)\right) \lambda+\omega_{1} \omega_{2}-S_{0}\left(\sigma f_{1}^{\prime}(0)+\omega_{2} f_{2}^{\prime}(0)\right)=0 .
$$

If $R_{0}<1$, one immediately gets $\omega_{1} \omega_{2}-S_{0}\left(\sigma f_{1}^{\prime}(0)+\omega_{2} f_{2}^{\prime}(0)\right)=\omega_{1} \omega_{2}\left(1-R_{0}\right)>0$, and by the expression of $R_{0}$, we have $S_{0} f_{2}^{\prime}(0)<\omega_{1}$, then $\omega_{1}+\omega_{2}-S_{0} f_{2}^{\prime}(0)>0$. Thus, by the Routh-Hurwitz criterion, the eigenvalues $\lambda_{j}(j=2,3)$ of $J_{E_{0}}$ have negative real part if $R_{0}<1$. If $R_{0}>1$, then $\omega_{1} \omega_{2}-S_{0}\left(\sigma f_{1}^{\prime}(0)+\omega_{2} f_{2}^{\prime}(0)\right)<0$, and this suggests that $J_{E_{0}}$ admits a positive real eigenvalue. Consequently, we have the following result.

Theorem 1 If $R_{0}<1$, then the disease-free equilibrium $E_{0}$ is locally asymptotically stable. $E_{0}$ is unstable if $R_{0}>1$.

Now, we analyze the local stability of the endemic equilibrium of the system (1) by assuming that $R_{0}>1$. The Jacobian matrix of system (1) at the equilibrium $E_{*}$ is as follows

$$
J_{E_{*}}=\left(\begin{array}{ccc}
-\mu-f_{1}\left(I^{*}\right)-f_{2}\left(E^{*}\right) & -S^{*} f_{2}^{\prime}\left(E^{*}\right) & -S^{*} f_{1}^{\prime}\left(I^{*}\right)+\gamma \\
f_{1}\left(I^{*}\right)+f_{2}\left(E^{*}\right) & S^{*} f_{2}^{\prime}\left(E^{*}\right)-\omega_{1} & S^{*} f_{1}^{\prime}\left(I^{*}\right) \\
0 & \sigma & -\omega_{2}
\end{array}\right) .
$$

Therefore, its characteristic equation at $E_{*}$ is

$$
\lambda^{3}+C_{2} \lambda^{2}+C_{1} \lambda+C_{0}=0,
$$

where

$$
\begin{aligned}
C_{2}= & \mu+\omega_{2}+f_{1}\left(I^{*}\right)+\frac{S^{*}}{E^{*}} f_{1}\left(I^{*}\right)+f_{2}\left(E^{*}\right)+S^{*}\left(\frac{f_{2}\left(E^{*}\right)}{E^{*}}-f_{2}^{\prime}\left(E^{*}\right)\right), \\
C_{1}= & \left(\omega_{1}+\omega_{2}\right)\left(f_{1}\left(I^{*}\right)+f_{2}\left(E^{*}\right)\right)+\mu\left(\omega_{2}+S^{*}\left(\frac{f_{2}\left(E^{*}\right)}{E^{*}}-f_{2}^{\prime}\left(E^{*}\right)\right)\right)+\sigma S^{*}\left(\frac{f_{1}\left(I^{*}\right)}{I^{*}}-f_{1}^{\prime}\left(I^{*}\right)\right) \\
& +\omega_{2} S^{*}\left(\frac{f_{2}\left(E^{*}\right)}{E^{*}}-f_{2}^{\prime}\left(E^{*}\right)\right), \\
C_{0}= & \left(\omega_{1} \omega_{2}-\sigma \gamma\right)\left(f_{1}\left(I^{*}\right)+f_{2}\left(E^{*}\right)\right)+\mu \sigma S^{*}\left(\frac{f_{1}\left(I^{*}\right)}{I^{*}}-f_{1}^{\prime}\left(I^{*}\right)\right)+\mu \omega_{2} S^{*}\left(\frac{f_{2}\left(E^{*}\right)}{E^{*}}-f_{2}^{\prime}\left(E^{*}\right)\right) .
\end{aligned}
$$


By $(H 2), \frac{f_{i}(x)}{x}$ is decreasing on $(0,+\infty)$, so $\left(\frac{f_{i}(x)}{x}\right)^{\prime} \leq 0$, which implies that $\frac{f_{i}(x)}{x}-f_{i}^{\prime}(x) \geq 0$ for all $x>0$. Further, we have $\omega_{1} \omega_{2}-\sigma \gamma=\mu(\mu+\delta+\gamma)+\sigma(\mu+\delta)>0$. Then it is easy to show that $C_{2}>0, C_{1}>0, C_{0}>0$ and $C_{2} C_{1}>C_{0}$. Thus, by the Routh-Hurwitz criterion, all roots $\lambda_{j}(j=1,2,3)$ of (7) have negative real part. Hence, we have the following theorem.

Theorem 2 When $R_{0}>1$, the endemic equilibrium $E_{*}$ is locally asymptotically stable.

\section{Global Stability}

The aim of this section is to analyze the global stability of the equilibria. First, we consider the global stability of model (1) at the disease-free equilibrium $E_{0}$.

Theorem 3 If $R_{0} \leq 1$, then the disease-free equilibrium $E_{0}$ is globally asymptotically stable.

Proof Let $U$ be the Lyapunov functional defined as

$$
U(t)=U_{0}(t)+U_{1}(t)
$$

where

$$
\begin{aligned}
& U_{0}(t)=S_{0} g\left(\frac{S}{S_{0}}\right)+E+\frac{\omega_{1} f_{1}^{\prime}(0)}{\sigma f_{1}^{\prime}(0)+\omega_{2} f_{2}^{\prime}(0)} I, \\
& U_{1}(t)=\frac{\gamma}{\sigma S_{0}} \frac{I^{2}}{2}+\frac{\gamma}{(2 \mu+\delta) S_{0}} \frac{\left(S-S_{0}+E+I\right)^{2}}{2}, \\
& g(x)=x-1-\ln x, x>0 .
\end{aligned}
$$

The derivatives of functions $U_{0}$ and $U_{1}$ along the solution of system (1) are

$$
\begin{aligned}
\frac{d U_{0}}{d t}= & \left(1-\frac{S_{0}}{S}\right)\left(\Lambda-\mu S-S f_{1}(I)-S f_{2}(E)+\gamma I\right)+\left(S f_{1}(I)+S f_{2}(E)-\omega_{1} E\right) \\
& \quad+\frac{\omega_{1} f_{1}^{\prime}(0)}{\sigma f_{1}^{\prime}(0)+\omega_{2} f_{2}^{\prime}(0)}\left(\sigma E-\omega_{2} I\right) \\
= & \frac{-\mu\left(S-S_{0}\right)^{2}}{S}+\left(f_{1}(I)+f_{2}(E)\right) S_{0} \\
& \quad-\frac{\omega_{1} \omega_{2}}{\sigma f_{1}^{\prime}(0)+\omega_{2} f_{2}^{\prime}(0)}\left(f_{1}^{\prime}(0) I+f_{2}^{\prime}(0) E\right)+\gamma I\left(1-\frac{S_{0}}{S}\right),
\end{aligned}
$$

and 


$$
\begin{aligned}
\frac{d U_{1}}{d t} & =\frac{\gamma}{\sigma S_{0}} I\left(\sigma E-\omega_{2} I\right)+\frac{\gamma}{(2 \mu+\delta) S_{0}}\left(-\mu\left(S-S_{0}+E\right)^{2}-(\mu+\delta) I^{2}-(2 \mu+\delta) I\left(S-S_{0}\right)-(2 \mu+\delta) E I\right) \\
& =-\frac{\gamma}{S_{0}}\left(\frac{\omega_{2}}{\sigma}+\frac{\mu+\delta}{2 \mu+\delta}\right) I^{2}-\frac{\mu \gamma}{(2 \mu+\delta) S_{0}}\left(S-S_{0}+E\right)^{2}-\gamma I\left(\frac{S}{S_{0}}-1\right) .
\end{aligned}
$$

Then

$$
\begin{aligned}
\frac{d U}{d t}= & \frac{-\mu\left(S-S_{0}\right)^{2}}{S}-\frac{\gamma}{S_{0}}\left(\frac{\omega_{2}}{\sigma}+\frac{\mu+\delta}{2 \mu+\delta}\right) I^{2}-\frac{\mu \gamma}{(2 \mu+\delta) S_{0}}\left(S-S_{0}+E\right)^{2}-\gamma I \frac{\left(S-S_{0}\right)^{2}}{S S_{0}} \\
& +\left(f_{1}(I)+f_{2}(E)\right) S_{0}-\frac{\omega_{1} \omega_{2}}{\sigma f_{1}^{\prime}(0)+\omega_{2} f_{2}^{\prime}(0)}\left(f_{1}^{\prime}(0) I+f_{2}^{\prime}(0) E\right) .
\end{aligned}
$$

According to (2), we have $f_{1}(I) \leq f_{1}^{\prime}(0) I$ and $f_{2}(E) \leq f_{2}^{\prime}(0) E$. Hence

$$
\begin{aligned}
\frac{d U}{d t} \leq & -\frac{\mu S_{0}+\gamma I}{S S_{0}}\left(S-S_{0}\right)^{2}-\frac{\gamma}{S_{0}}\left(\frac{\omega_{2}}{\sigma}+\frac{\mu+\delta}{2 \mu+\delta}\right) I^{2} \\
& -\frac{\mu \gamma}{(2 \mu+\delta) S_{0}}\left(S-S_{0}+E\right)^{2}+\left(f_{1}^{\prime}(0) I+f_{2}^{\prime}(0) E\right) S_{0}\left(1-\frac{1}{R_{0}}\right) .
\end{aligned}
$$

Therefore, $R_{0} \leq 1$ ensures that $\frac{d U}{d t} \leq 0$. Furthermore, it is easy to verify that the singleton $\left\{E_{0}\right\}$ is the largest compact invariant set in $\left\{(S, E, I) \in \mathbb{R}_{+}^{3}: \frac{d U}{d t}=0\right\}$. By LaSalle's invariance principle [32], we get that equilibrium $E_{0}$ of system (1) is globally asymptotically stable if $R_{0} \leq 1$.

In the following theorem, we study the global stability of the endemic equilibrium $E_{*}$.

Theorem 4 Assume that $R_{0}>1$. The endemic equilibrium $E_{*}$ is globally asymptotically stable if

$$
\mu S^{*}-\gamma I^{*} \geq 0
$$

Proof Let $V$ be the Lyapunov functional defined as

$$
V(t)=V_{0}(t)+V_{1}(t)
$$

where

$$
V_{0}(t)=S^{*} g\left(\frac{S}{S^{*}}\right)+E^{*} g\left(\frac{E}{E^{*}}\right)+\frac{S^{*} f_{1}\left(I^{*}\right)}{\sigma E^{*}} I^{*} g\left(\frac{I}{I^{*}}\right),
$$

and

$$
V_{1}(t)=\frac{\gamma}{\sigma S^{*}} \frac{\left(I-I^{*}\right)^{2}}{2}+\frac{\gamma}{(2 \mu+\delta) S^{*}} \frac{\left(S-S^{*}+E-E^{*}+I-I^{*}\right)^{2}}{2} .
$$

By means of the equalities

$$
\Lambda=\mu S^{*}+S^{*} f_{1}\left(I^{*}\right)+S^{*} f_{2}\left(E^{*}\right)-\gamma I^{*}, S^{*} f_{1}\left(I^{*}\right)+S^{*} f_{2}\left(E^{*}\right)=\omega_{1} E^{*}, \omega_{2}=\frac{\sigma E^{*}}{I^{*}},
$$

the derivative of $V_{0}$ is 


$$
\begin{aligned}
\frac{d V_{0}}{d t}= & \left(1-\frac{S^{*}}{S}\right)\left(-\mu\left(S-S^{*}\right)+S^{*} f_{1}\left(I^{*}\right)-S f_{1}(I)+S^{*} f_{2}\left(E^{*}\right)-S f_{2}(E)+\gamma\left(I-I^{*}\right)\right) \\
& +\left(1-\frac{E^{*}}{E}\right)\left(S f_{1}(I)-S^{*} f_{1}\left(I^{*}\right) \frac{E}{E^{*}}+S f_{2}(E)-S^{*} f_{2}\left(E^{*}\right) \frac{E}{E^{*}}\right)+S^{*} f_{1}\left(I^{*}\right)\left(1-\frac{I^{*}}{I}\right)\left(\frac{E}{E^{*}}-\frac{I}{I^{*}}\right) \\
= & -\mu \frac{\left(S-S^{*}\right)^{2}}{S}+S^{*} f_{1}\left(I^{*}\right)\left(1-\frac{S^{*}}{S}-\frac{S f_{1}(I)}{S^{*} f_{1}\left(I^{*}\right)}+\frac{f_{1}(I)}{f_{1}\left(I^{*}\right)}\right)+S^{*} f_{2}\left(E^{*}\right)\left(1-\frac{S^{*}}{S}-\frac{S f_{2}(E)}{S^{*} f_{2}\left(E^{*}\right)}+\frac{f_{2}(E)}{f_{2}\left(E^{*}\right)}\right) \\
& +\gamma\left(I-I^{*}\right)\left(1-\frac{S^{*}}{S}\right)+S^{*} f_{1}\left(I^{*}\right)\left(1-\frac{E}{E^{*}}-\frac{S E^{*} f_{1}(I)}{S^{*} E f_{1}\left(I^{*}\right)}+\frac{S f_{1}(I)}{S^{*} f_{1}\left(I^{*}\right)}\right) \\
& +S^{*} f_{2}\left(E^{*}\right)\left(1-\frac{E}{E^{*}}-\frac{S E^{*} f_{2}(E)}{S^{*} E f_{2}\left(E^{*}\right)}+\frac{S f_{2}(E)}{S^{*} f_{2}\left(E^{*}\right)}\right)+S^{*} f_{1}\left(I^{*}\right)\left(\frac{E}{E^{*}}-\frac{I}{I^{*}}-\frac{I^{*} E}{I E^{*}}+1\right) \\
= & -\mu \frac{\left(S-S^{*}\right)^{2}}{S}+S^{*} f_{1}\left(I^{*}\right)\left(3-\frac{S^{*}}{S}-\frac{S E^{*} f_{1}(I)}{S^{*} E f_{1}\left(I^{*}\right)}-\frac{I}{I^{*}}-\frac{I^{*} E}{I E^{*}}+\frac{f_{1}(I)}{f_{1}\left(I^{*}\right)}\right) \\
& +S^{*} f_{2}\left(E^{*}\right)\left(2-\frac{S^{*}}{S}-\frac{E}{E^{*}}-\frac{S E^{*} f_{2}(E)}{S^{*} E f_{2}\left(E^{*}\right)}+\frac{f_{2}(E)}{f_{2}\left(E^{*}\right)}\right)+\gamma\left(I-I^{*}\right)\left(1-\frac{S^{*}}{S}\right) \\
= & -\mu \frac{\left(S-S^{*}\right)^{2}}{S}+S^{*} f_{1}\left(I^{*}\right)\left(\left(\frac{f_{1}(I)}{f_{1}\left(I^{*}\right)}-1-\frac{I}{I^{*}}+\frac{I f_{1}\left(I^{*}\right)}{I^{*} f_{1}(I)}\right)+\left(4-\frac{S^{*}}{S}-\frac{S E^{*} f_{1}(I)}{S^{*} E f_{1}\left(I^{*}\right)}-\frac{I^{*} E}{I E^{*}}-\frac{I f_{1}\left(I^{*}\right)}{I^{*} f_{1}(I)}\right)\right) \\
& +S^{*} f_{2}\left(E^{*}\right)\left(\left(\frac{f_{2}(E)}{f_{2}\left(E^{*}\right)}-1-\frac{E}{E^{*}}+\frac{E f_{2}\left(E^{*}\right)}{E^{*} f_{2}(E)}\right)+\left(3-\frac{S^{*}}{S}-\frac{S E^{*} f_{2}(E)}{S^{*} E f_{2}\left(E^{*}\right)}-\frac{E f_{2}\left(E^{*}\right)}{E^{*} f_{2}(E)}\right)\right)+\gamma\left(I-I^{*}\right)\left(1-\frac{S^{*}}{S}\right) .
\end{aligned}
$$

The derivative of $V_{1}$ is

$$
\begin{aligned}
\frac{d V_{1}}{d t}= & \frac{\gamma}{S^{*}}\left(I-I^{*}\right)\left(E-E^{*}\right)-\frac{\gamma}{\sigma S^{*}}(\mu+\delta+\gamma)\left(I-I^{*}\right)^{2}+\frac{\gamma}{(2 \mu+\delta) S^{*}}\left(-\mu\left(S-S^{*}+E-E^{*}\right)^{2}\right. \\
& \left.-(\mu+\delta)\left(I-I^{*}\right)^{2}-(2 \mu+\delta)\left(S-S^{*}\right)\left(I-I^{*}\right)-(2 \mu+\delta)\left(I-I^{*}\right)\left(E-E^{*}\right)\right) \\
= & -\frac{\gamma}{\sigma S^{*}}(\mu+\delta+\gamma)\left(I-I^{*}\right)^{2}-\frac{\mu \gamma}{(2 \mu+\delta) S^{*}}\left(S-S^{*}+E-E^{*}\right)^{2}-\frac{\gamma(\mu+\delta)}{(2 \mu+\delta) S^{*}}\left(I-I^{*}\right)^{2}-\gamma\left(\frac{S}{S^{*}}-1\right)\left(I-I^{*}\right) \\
= & -\frac{\gamma}{S^{*}}\left(\frac{\mu+\delta+\gamma}{\sigma}+\frac{\mu+\delta}{2 \mu+\delta}\right)\left(I-I^{*}\right)^{2}-\frac{\mu \gamma}{(2 \mu+\delta) S^{*}}\left(S-S^{*}+E-E^{*}\right)^{2}-\gamma\left(\frac{S}{S^{*}}-1\right)\left(I-I^{*}\right) .
\end{aligned}
$$

Then the sum of these two functions gives

$$
\begin{aligned}
\frac{d V}{d t}= & -\left(\left(\mu S^{*}-\gamma I^{*}\right)+\gamma I\right) \frac{\left(S-S^{*}\right)^{2}}{S}-\frac{\gamma}{S^{*}}\left(\frac{\mu+\delta+\gamma}{\sigma}+\frac{\mu+\delta}{2 \mu+\delta}\right)\left(I-I^{*}\right)^{2}-\frac{\mu \gamma}{(2 \mu+\delta) S^{*}}\left(S-S^{*}+E-E^{*}\right)^{2} \\
& +S^{*} f_{1}\left(I^{*}\right)\left(\left(\frac{f_{1}(I)}{f_{1}\left(I^{*}\right)}-1-\frac{I}{I^{*}}+\frac{I f_{1}\left(I^{*}\right)}{I^{*} f_{1}(I)}\right)+\left(4-\frac{S^{*}}{S}-\frac{S E^{*} f_{1}(I)}{S^{*} E f_{1}\left(I^{*}\right)}-\frac{I^{*} E}{I E^{*}}-\frac{I f_{1}\left(I^{*}\right)}{I^{*} f_{1}(I)}\right)\right) \\
& +S^{*} f_{2}\left(E^{*}\right)\left(\left(\frac{f_{2}(E)}{f_{2}\left(E^{*}\right)}-1-\frac{E}{E^{*}}+\frac{E f_{2}\left(E^{*}\right)}{E^{*} f_{2}(E)}\right)+\left(3-\frac{S^{*}}{S}-\frac{S E^{*} f_{2}(E)}{S^{*} E f_{2}\left(E^{*}\right)}-\frac{E f_{2}\left(E^{*}\right)}{E^{*} f_{2}(E)}\right)\right) .
\end{aligned}
$$

By the hypotheses $(H 1)$ and $(H 2)$, we obtain

$$
\begin{gathered}
\frac{f_{1}(I)}{f_{1}\left(I^{*}\right)}-1-\frac{I}{I^{*}}+\frac{I f_{1}\left(I^{*}\right)}{I^{*} f_{1}(I)}=\frac{1}{I^{*}}\left(\frac{I^{*}}{f_{1}\left(I^{*}\right)}-\frac{I}{f_{1}(I)}\right)\left(f_{1}(I)-f_{1}\left(I^{*}\right)\right) \leq 0, \\
\frac{f_{2}(E)}{f_{2}\left(E^{*}\right)}-1-\frac{E}{E^{*}}+\frac{E f_{2}\left(E^{*}\right)}{E^{*} f_{2}(E)}=\frac{1}{E^{*}}\left(\frac{E^{*}}{f_{2}\left(E^{*}\right)}-\frac{E}{f_{2}(E)}\right)\left(f_{2}(E)-f_{2}\left(E^{*}\right)\right) \leq 0,
\end{gathered}
$$

and using the arithmetic-geometric inequality, we have

$$
\begin{array}{r}
4-\frac{S^{*}}{S}-\frac{S E^{*} f_{1}(I)}{S^{*} E f_{1}\left(I^{*}\right)}-\frac{I^{*} E}{I E^{*}}-\frac{I f_{1}\left(I^{*}\right)}{I^{*} f_{1}(I)} \leq 0, \\
3-\frac{S^{*}}{S}-\frac{S E^{*} f_{2}(E)}{S^{*} E f_{2}\left(E^{*}\right)}-\frac{E f_{2}\left(E^{*}\right)}{E^{*} f_{2}(E)} \leq 0 .
\end{array}
$$


Hence, the condition (8) ensures that $\frac{d V}{d t} \leq 0$. Further, the largest invariant set of $\left\{(S, E, I) \in \mathbb{R}_{+}^{3}: \frac{d V}{d t}=0\right\}$ is the singleton $\left\{E_{*}\right\}$. By applying the LaSalle's invariance principle, we can obtain that the endemic equilibrium $E_{*}$ of model (1) is globally asymptotically stable if $R_{0}>1$ and $\mu S^{*}-\gamma I^{*} \geq 0$.

Remark 1 When $\gamma=0$, the system (1) reduces to an SEI model, and the endemic equilibrium $E_{*}$ is globally asymptotically stable if $R_{0}>1$.

Corollary 1 For $\gamma>0$, the endemic equilibrium $E_{*}$ is globally asymptotically stable if

$$
1<R_{0} \leq 1+\frac{\phi}{\gamma}
$$

where $\phi$ given in the proof of Lemma 1.

Proof We prove that the condition (8) in Theorem 4 holds if the condition $R_{0} \leq 1+\frac{\phi}{\gamma}$ holds. We have $I^{*}$ satisfies the following

$$
\begin{aligned}
I^{*} & =\frac{1}{\phi}\left(\Lambda-\frac{\mu \omega_{1} \omega_{2}}{\sigma} \frac{1}{\frac{f_{1}\left(I^{*}\right)}{I^{*}}+\frac{f_{2}\left(\frac{\omega_{2}}{\sigma} I^{*}\right)}{I^{*}}}\right) \leq \frac{1}{\phi}\left(\Lambda-\frac{\mu \omega_{1} \omega_{2}}{\sigma} \frac{1}{f_{1}^{\prime}(0)+\frac{\omega_{2}}{\sigma} f_{2}^{\prime}(0)}\right) \\
& =\frac{\mu \omega_{1} \omega_{2}}{\phi\left(\sigma f_{1}^{\prime}(0)+\omega_{2} f_{2}^{\prime}(0)\right)}\left(R_{0}-1\right) .
\end{aligned}
$$

Therefore, we obtain that

$$
\begin{aligned}
\mu S^{*}-\gamma I^{*}= & \frac{\frac{\mu \omega_{1} \omega_{2} I^{*}}{\sigma}}{f_{1}\left(I^{*}\right)+f_{2}\left(\frac{\omega_{2}}{\sigma} I^{*}\right)}-\gamma I^{*} \\
= & \frac{\frac{\mu \omega_{1} \omega_{2}}{\sigma} I^{*}}{f_{1}\left(I^{*}\right)+f_{2}\left(\frac{\omega_{2}}{\sigma} I^{*}\right)}\left(1-\gamma \frac{\sigma}{\mu \omega_{1} \omega_{2}}\left(f_{1}\left(I^{*}\right)+f_{2}\left(\frac{\omega_{2}}{\sigma} I^{*}\right)\right)\right) \\
& \geq \frac{\frac{\mu \omega_{1} \omega_{2}}{\sigma} I^{*}}{f_{1}\left(I^{*}\right)+f_{2}\left(\frac{\omega_{2}}{\sigma} I^{*}\right)}\left(1-\gamma \frac{\sigma f_{1}^{\prime}(0)+\omega_{2} f_{2}^{\prime}(0)}{\mu \omega_{1} \omega_{2}} I^{*}\right) \\
& \geq \frac{\frac{\mu \omega_{1} \omega_{2}}{\sigma} I^{*}}{f_{1}\left(I^{*}\right)+f_{2}\left(\frac{\omega_{2}}{\sigma} I^{*}\right)}\left(1-\frac{\gamma}{\phi}\left(R_{0}-1\right)\right)
\end{aligned}
$$

which completes the proof.

\section{Application and Numerical Simulations}

The main purpose of this section is to give a particular nonlinear incidence rate to illustrate our main results. Consider the system 
Table 1 Parameter values for model (9)

\begin{tabular}{llllllllll}
\hline Parameter & $\Lambda$ & $\mu$ & $\beta_{1}$ & $\beta_{2}$ & $\sigma$ & $\delta$ & $\gamma$ & $\alpha_{1}$ & $\alpha_{2}$ \\
\hline Value & 130 & 0.0939 & 0.00015 & 0.0002 & 0.6 & 0.037 & 0.8 & 0.2 & 0.1 \\
\hline
\end{tabular}

Table 2 Parameter values for model (9)

\begin{tabular}{llllllllll}
\hline Parameter & $\Lambda$ & $\mu$ & $\beta_{1}$ & $\beta_{2}$ & $\sigma$ & $\delta$ & $\gamma$ & $\alpha_{1}$ & $\alpha_{2}$ \\
\hline Value & 130 & 0.0939 & 0.001 & 0.0015 & 0.6 & 0.037 & 0.8 & 0.2 & 0.1 \\
\hline
\end{tabular}

$$
\left\{\begin{array}{l}
\frac{d S(t)}{d t}=\Lambda-\mu S(t)-\frac{\beta_{1} S(t) I(t)}{1+\alpha_{1} I(t)}-\frac{\beta_{2} S(t) E(t)}{1+\alpha_{2} E(t)}+\gamma I(t), \\
\frac{d E(t)}{d t}=\frac{\beta_{1} S(t) I(t)}{1+\alpha_{1} I(t)}+\frac{\beta_{2} S(t) E(t)}{1+\alpha_{2} E(t)}-(\mu+\sigma) E(t), \\
\frac{d I(t)}{d t}=\sigma E(t)-(\mu+\delta+\gamma) I(t),
\end{array}\right.
$$

subject to initial conditions

$$
S(0) \geq 0, E(0) \geq 0, I(0) \geq 0,
$$

where $\beta_{1}$ and $\beta_{2}$ are the rates of the efficient contact in infected period and latent period, respectively. The positive constants $\alpha_{1}$ and $\alpha_{2}$ measure the saturation or inhibitory effect. The remaining parameters have the same biological significance as those in system (1). Model (9) is a particular case of system (1) by choosing $f_{1}(I)=\frac{\beta_{1} I}{1+\alpha_{1} I}$ and $f_{2}(E)=\frac{\beta_{2} E}{1+\alpha_{2} E}$.

Obviously, the hypotheses $(H 1)-(H 2)$ are satisfied. The basic reproduction number of model (9) is

$$
R_{0}=\frac{\sigma \beta_{1} \Lambda}{\mu(\mu+\sigma)(\mu+\delta+\gamma)}+\frac{\beta_{2} \Lambda}{\mu(\mu+\sigma)} .
$$

Now, we give some numerical simulations in order to validate our theoretical results. Firstly, we simulate model (9) with the parameter values as shown in Table 1.

By calculation, we have $R_{0}=0.5919 \leq 1$. Hence, by Lemma 1, model (9) has only an disease-free equilibrium $E_{0}=(1384.45,0,0)$. Fig. 1 shows that the trajectories of $S(t)$, $E(t)$ and $I(t)$, which are the solutions of (9), from different initial values to $E_{0}$. This supports the analytical result obtained in Theorem 3, i.e., the disease-free equilibrium $E_{0}$ is globally asymptotically stable provided $R_{0} \leq 1$. Thus, under the condition $R_{0} \leq 1$, the disease will be eradicated.

Now, we simulate the case when the basic reproduction number is bigger than one. For this end, we consider model (9) with the parameter values as shown in Table 2.

By computing, we get $R_{0}=4.2787>1$ and $\mu S^{*}-\gamma I^{*}=97.0392 \geq 0$. Fig. 2 shows that the trajectories of $S(t), E(t)$ and $I(t)$, which are the solutions of (9), from different initial values to $E_{*}=(1192.7,29.0039,18.6941)$. This confirms the global stability of 

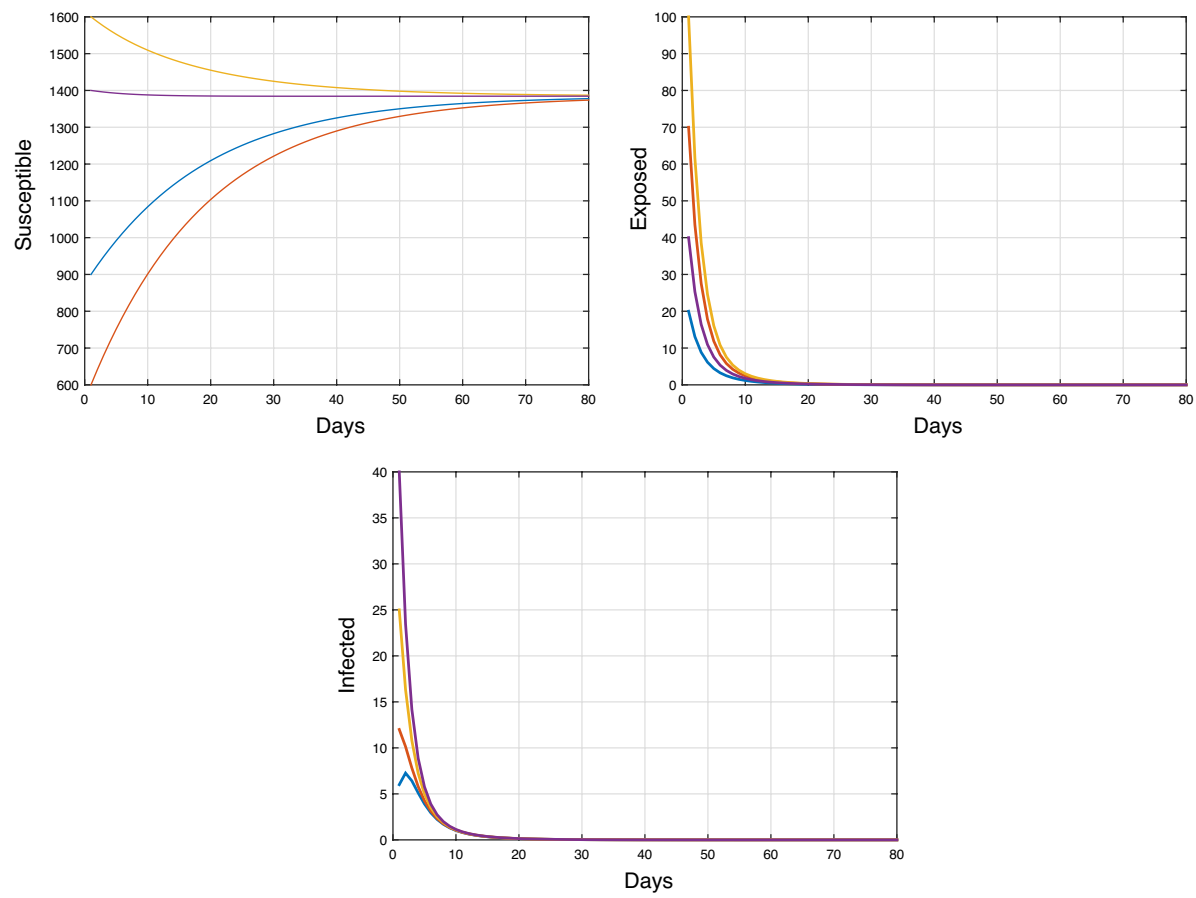

Fig. 1 Plot of the time evolution of $S(t), E(t)$ and $I(t)$, which are the solutions of (9). The parameter values are tabulated in Table 1 . This plot shows the stability of the disease-free equilibrium $E_{0}$ from different values

the endemic equilibrium $E_{*}$ and thus supports the theoretical result given in Theorem 4, which means that the disease becomes endemic.

Next, we provide a numerical simulation using real data for the transmission of COVID-19 in Morocco during the year 2020 [33]. On 27 October, we have $S(0)=37055403$ [34], $I(0)=203733$ [33], then we assume $E(0)=400000$. Fig. 3 shows that the trajectories of $S(t), E(t)$ and $I(t)$ in the model under investigation (9), for the parameter values as shown in Table 3 , converges towards the endemic equilibrium $E_{*}=\left(1.9007 \times 10^{7}, 1.8539 \times 10^{6}, 2.3607 \times 10^{6}\right)$.

In this case, we have the basic reproduction number is greater than unity $R_{0}=1.5159>1$ and $\mu S^{*}-\gamma I^{*} \geq 0$, which means that the disease persists. In this situation, it will be important to eventually undertake some strategies like quarantine, isolation, wearing of masks, disinfection and if it will be possible vaccination.

\section{Conclusion}

This paper presents a mathematical study on the dynamical behavior of an SEIS epidemic model with infectivity in incubation period and general incidence rates. From the system, we have found the following basic reproduction number 

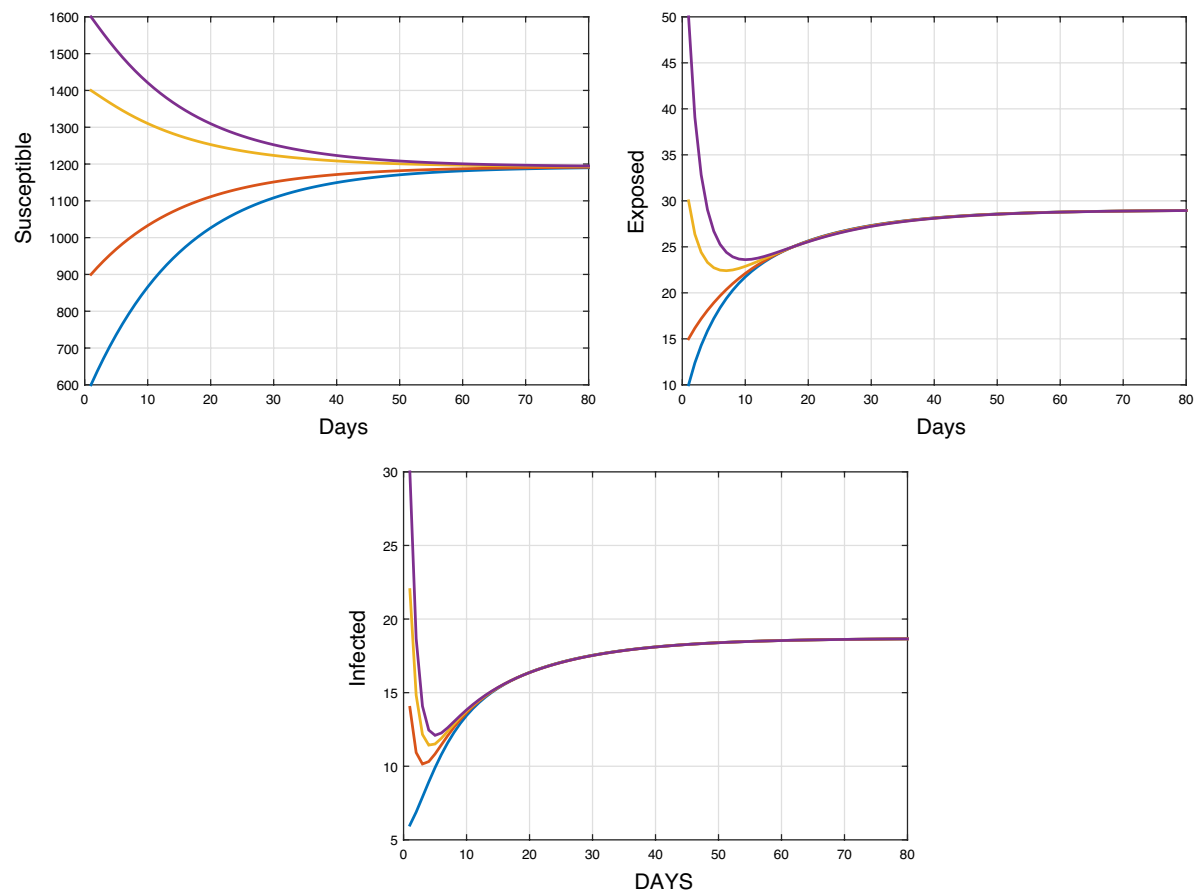

Fig. 2 Plot of the time evolution of $S(t), E(t)$ and $I(t)$, which are the solutions of (9). The parameter values are tabulated in Table 2. This plot shows the stability of the endemic equilibrium $E_{*}$ from different values

Table 3 Parameter values for model (9)

\begin{tabular}{llllllllll}
\hline Parameter & $\Lambda$ & $\mu$ & $\beta_{1}$ & $\beta_{2}$ & $\sigma$ & $\delta$ & $\gamma$ & $\alpha_{1}$ & $\alpha_{2}$ \\
\hline Value & 278.15 & 0.05861 & 0.000079 & 0.0001 & 0.5 & 0.017 & 0.428 & 0.14 & 0.145 \\
\hline
\end{tabular}

$$
R_{0}=\frac{\sigma \Lambda f_{1}^{\prime}(0)}{\mu(\mu+\sigma)(\mu+\delta+\gamma)}+\frac{\Lambda f_{2}^{\prime}(0)}{\mu(\mu+\sigma)} .
$$

It determines whether the disease will die out or persist in the population as time increases. We have shown that if $R_{0} \leq 1$, the disease-free equilibrium $E_{0}$ is globally asymptotically, which means that the disease cannot persist in the population, and the situation is under control. Moreover, we have proved that if $R_{0}>1$, the disease-free equilibrium becomes unstable and the model has an endemic equilibrium $E_{*}$ which is globally asymptotically stable provided that $\mu S^{*} \geq \gamma I^{*}$, so that the disease, if initially present, will be persistent at the unique endemic equilibrium level. An application and some numerical simulations are presented to verify the feasibility of the theoretical results.

We would like to point out here that Theorem 4 leaves us an open problem: whether the endemic equilibrium $E_{*}$ is globally asymptotically stable when $R_{0}>1$ without the additional condition $\mu S^{*}-\gamma I^{*} \geq 0$ ?. We leave this for future work. 

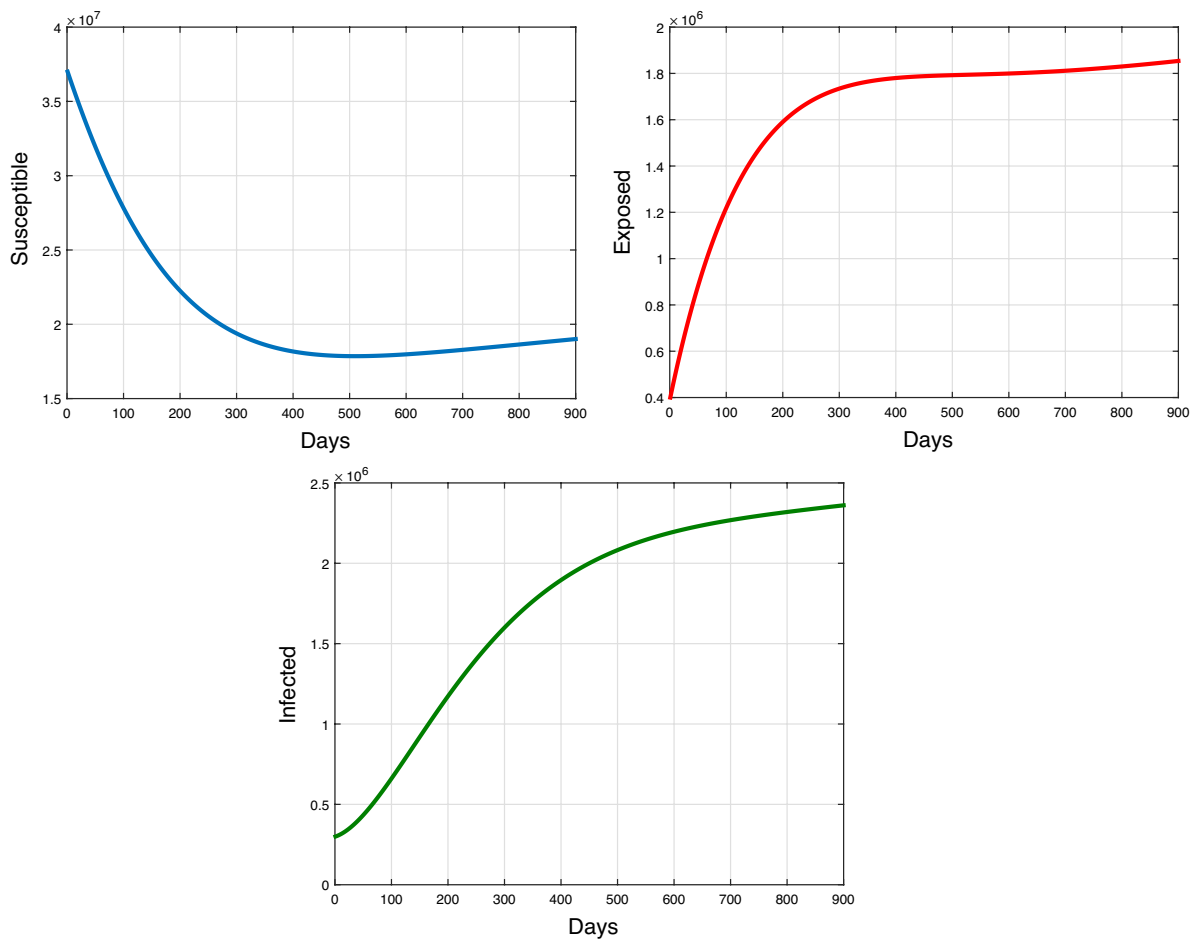

Fig. 3 Plot of the time evolution of $S(t), E(t)$ and $I(t)$, which are the solutions of (9). The parameter values are tabulated in Table 3. This plot shows the dynamics of COVID-19 in Morocco

\section{Declarations}

Conflict of Interest The authors declare that they have no competing interests.

\section{References}

1. Gorbalenya, A.E., Baker, S.C., Baric, R.S., de Groot, R.J., Drosten, C., Gulyaeva, A.A. et al.: The species severe acute respiratory syndromerelated coronavirus: classifying 2019-nCoV and naming it SARS-CoV-2 . Nat. Microbiol. 5, 536-544 (2020)

2. Li, Q., Guan, X., Wu, P., et al.: Early transmission dynamics in Wuhan, China, of novel coronavirusinfected pneumonia. N. Engl. J. Med. (2020). https://doi.org/10.1056/NEJMoa2001316

3. Zhang, J., et al.: Global analysis of SEI epidemic model with the constant inflows of different compartments. J. Xi'an Jiaotong Univ. 6, 653-656 (2003)

4. Wang, L., Li, J.: Qualitative analysis of a SEIS epidemic model with nonlinear incidence rate. Appl. Math. Phys. 27(5), 592-596 (2006)

5. Abta, A., Kaddar, A., Alaoui, H.T.: Global stability for delay SIR and SEIR epidemic models with saturated incidence rates. Electron. J. Differ. Equ. 386(2), 956-965 (2012)

6. $\mathrm{Xu}, \mathrm{R} .:$ Global dynamics of an SEIRI epidemiological model with time delay. Appl. Math. Comput. 232(1), 436-444 (2014)

7. Singh, P., Srivastava, S.K., Arora, U.: Stability of SEIR model of infectious diseases with human immunity. Glob. J. Pure Appl. Math. 6, 1811-1819 (2017)

8. Lv, G., Lu, Z.: Global asymptotic stability for the SEIRS models with varying total population size. Math. Biosci. 296, 17-25 (2018) 
9. National health commission of the people's Republic of China (2020). http://www.nhc.gov.cn/. Accessed March 2020

10. Yuang, S.L., et al.: A kind of epidemic model having infectious force in both latent period and infected period. J. Biomath. 16, 392-398 (2001)

11. Guihua, L., Zhen, J.: Global stability of an SEI epidemic model. Chaos Solitions Fractals 21, 925-931 (2004)

12. Guihua, L., Zhen, J.: Global stability of an SEI epidemic model with general contact rate. Chaos Solitions Fractals 23, 997-1004 (2005)

13. Mukhopadhyay, B., Bhattacharyya, R.: Analysis of a spatially extended nonlinear SEIS epidemic model with distinct incidence for exposed and infectives. Nonlinear Anal. Real World Appl. 9(2), 585$598(2008)$

14. Smith, H.L.: Systems of ordinary differential equations which generate an order preserving flow. SIAM Rev. 30, 87-113 (1988)

15. Li, M.Y., Muldowney, J.S.: A geometric approach to global stability problems. SIAM J. Math. Anal. 27, 1070-1083 (1996)

16. Anderson, R.M., May, R.M.: Infectious Diseases of Humans: Dynamics and Control. Oxford University Press, Oxford (1991)

17. Hethcote, H.W.: The mathematics of infectious diseases. SIAM Rev. 42, 599-653 (2000)

18. Vargas-De-León, C.: On the global stability of SIS, SIR and SIRS epidemic models with standard incidence. Chaos Solitons Fractals 44, 1106-1110 (2011)

19. Capasso, V., Serio, G.: A generalization of the Kermack-Mckendrick deterministic epidemic model. Math. Biosci. 42, 43-61 (1978)

20. Huang, G., Takeuchi, Y., Ma, W., Wei, D.: Global stability for delay SIR and SEIR epidemic models with nonlinear incidence rate. Bull. Math. Biol. 72(5), 1192-1207 (2010)

21. Enatsu, Y., Nakata, Y., Muroya, Y.: Lyapunov functional techniques for the global stability analysis of a delayed SIRS epidemic model. Nonlinear Anal. RWA 13, 2120-2133 (2012)

22. Sigdel, R.P., McCluskey, C.C.: Global stability for an SEI model of infectious disease with immigration. Appl. Math. Comput. 243, 684-689 (2014)

23. Li, J., Yang, Y., Xiao, Y., Liu, S.: A class of Lyapunov functions and the global stability of some epidemic models with nonlinear incidence. J. Appl. Anal. Comput. 6, 38-46 (2016)

24. Li, T., Zhang, F., Liu, H., Chen, Y.: Threshold dynamics of an SIRS model with nonlinear incidence rate and transfer from infectious to susceptible. Appl. Math. Lett. 70, 52-57 (2017)

25. Wang, L., Zhang, X., Liu, Z.: An SEIR epidemic model with relapse and general nonlinear incidence rate with application to media impact. Qual. Theory Dyn. Syst. 17, 1-21 (2017)

26. Naim, M., Lahmidi, F., Namir, A.: Stability analysis of a delayed fractional order SIRS epidemic model with nonlinear incidence rate. Int. J. Appl. Math. 32(5), 733-745 (2019)

27. Lahrouz, A., Omari, L., Kiouach, D., Belmaâtic, A.: Complete global stability for an SIRS epidemic model with generalized non-linear incidence and vaccination. Appl. Math. Comput. 218(11), 65196525 (2012)

28. Wang, L., Li, Y., Pang, L.: Dynamics analysis of an epidemiological model with media impact and two delays. Math. Probl. Eng. 2016, Art. ID 1598932 (2016)

29. Wang, L., Liu, Z., Zhang, X.: Global dynamics for an age-structured epidemic model with media impact and incomplete vaccination. Nonlinear Anal. Real World Appl. 32, 136-158 (2016)

30. van den Driessche, P., Watmough, I.: Reproduction numbers and sub-threshold endemic equilibria for compartmental models of disease transmission. Math. Biosci. 180(1), 29-48 (2002)

31. Diekman, O., Heesterbeek, J.A.P.: Mathematical Epidemiology of Infectious Diseases: Model Building. Analysis and Interpretation. Wiley, New York (2000)

32. LaSalle, J.P.: The Stability of Dynamical Systems. Ser. Regional Conference Series in Applied Mathematics. Society for Industrial and Applied Mathematics, no. 25 (1976)

33. Ministry of Health, Morocco, Department of Epidemiology and Disease Control. http://www.sante. gov.ma/Pages/Accueil.aspx. Accessed 27 Oct 2020

34. https://www.worldometers.info/world-population/morocco-population/. Accessed 27 Oct 2020

Publisher's Note Springer Nature remains neutral with regard to jurisdictional claims in published maps and institutional affiliations. 